\title{
Time reversal in classical electromagnetism
}

\author{
Frank Arntzenius and Hilary Greaves*
}

February 7, 2007

\section{DRAFT - COMMENTS ARE VERY WELCOME.}

\begin{abstract}
Richard Feynman has claimed that anti-particles are nothing but particles 'propagating backwards in time'; that time reversing a particle state always turns it into the corresponding anti-particle state. According to standard quantum field theory textbooks this is not so: time reversal does not turn particles into anti-particles. Feynman's view is interesting because, in particular, it suggests a nonstandard, and possibly illuminating, interpretation of the CPT theorem.

In this paper, we explore a classical analog of Feynman's view, in the context of the recent debate between David Albert and David Malament over time reversal in classical electromagnetism.
\end{abstract}

\section{Introduction}

In the context of quantum electrodynamics, Feynman writes:

A backwards-moving electron when viewed with time moving forwards appears the same as an ordinary electron, except it's attracted to normal electrons - we say it has positive charge. For this reason it's called a 'positron'. The positron is a sister to the electron, and it is an example of an 'anti-particle'. This phenomenon is quite general. Every particle in Nature has an amplitude to move backwards in time, and therefore has an anti-particle. (Feynman, 1985):98

Department of Philosophy, Rutgers University, 26 Nichol Avenue, New Brunswick, NJ 08901-2882, USA. Email: arntzeni@rci.rutgers.edu; hgreaves@rci.rutgers.edu. 
Note that Feynman is not making any claims about backwards causation. He is merely claiming that if you time reverse a sequence of particle states you get a sequence of corresponding anti-particle states. Or, at least, that is the view that we are interested in comparing to the standard view, and that is the view we will call 'Feynman's view'.

Meanwhile, in classical electromagnetism: David Albert (Albert, 2000) has argued that classical electromagnetism is not time reversal invariant, because (according to him) there is no justification for flipping the sign of the magnetic field under time reversal. David Malament (Malament, 2004) has replied in defense of the standard view of time reversal, according to which the $\mathbf{B}$ field does flip sign and the theory is time reversal invariant.

Malament's discussion may leave one with the feeling that one only has to appreciate both (i) the four-dimensional formulation of classical electromagnetism and (ii) what we mean, or ought to mean, by 'time reversal', and the standard transformation $\mathbf{B} \stackrel{T}{\longmapsto}-\mathbf{B}$ will follow. This, however, is incorrect: there is an alternative to Malament's account, consistent with both (i) and (ii). It is an account according to which the magnetic field does not flip sign under time reversal (the electric field does), but the theory is time reversal invariant anyway; it is the classical analog of Feynman's view.

This paper explores the 'classical Feynman' view, and issues that it throws up concerning the ontology of electromagnetism and the meaning of time reversal.

The structure of the paper is as follows. In section 2 we discuss what time reversal is, and why one should care about it. Section 3 is a critical review of the existing debate concerning time reversal in classical electromagnetism: the standard 'textbook' account, Albert's objection, and Malament's reply. In section 4 we articulate the 'Feynman' account. Section 5 investigates the possibility of regarding the 'Malament' and 'Feynman' accounts as equivalent descriptions of the same underlying reality. Section 6 is the conclusion.

\section{Time reversal and the direction of time}

Let's start with the more-or-less standard account of what time reversal is, and why one should be interested in it.

Suppose we describe a world (or part of a world) using some set of coordinates $x, y, z, t$. A passive time reversal is what happens to this description when we describe the same world but instead use coordinates $x, y, z, t^{\prime}$ where $t^{\prime}=-t$. An active time reversal is the following: keep using the same coordinates, but change the world in such a way that the description of the world in these coordinates changes exactly as it does in the corresponding passive time reversal. 
(So active and passive time reversal have exactly the same effect on the coordinate dependent descriptions of worlds.)

Suppose now that we have a theory which is stated in terms of coordinate dependent descriptions of the world, i.e. a theory which says that only certain coordinate dependent descriptions describe physically possible worlds. Such a theory is said to be time reversal invariant iff time reversal turns solutions into solutions and non-solutions into non-solutions. (Since active and passive time reversals have the same effect on the coordinate dependent descriptions of worlds, it follows that coordinate dependent theories will be invariant under active time reversal iff they are invariant under active time reversal.)

Why might one be interested in the time reversal invariance of theories? Because failure of time reversal invariance of a theory indicates that time has an objective direction according to that theory. Why believe that? Well, suppose that we start with a coordinate dependent description of a world (or part of a world) which our theory allows. And suppose that after we do a passive coordinate transformation our theory says that the new (coordinate dependent) description of this world is no longer allowed. This seems odd: it's the same world after all, just described using one set of coordinates rather than another. How could the one be allowed by our theory and the other not? Indeed, this does not make much sense unless one supposes that the theory, as stated in coordinate dependent form, was true in the original coordinates but not in the new coordinates. And that means that according to the theory there is some objective difference between the $x, y, z, t$ coordinates and the $x, y, z, t^{\prime}$ coordinates (where $t^{\prime}=-t$ ). So time has an objective direction. And if we want to write our theory in a coordinate independent way we are going to have to introduce a representation of this temporal orientation into our formalism.

Let's now clarify and modify this standard account a little bit. Let's start by asking a question that is rarely asked in physics texts, namely, what determines how things transform under a time reversal transformation? Well, space-time has some coordinate independent structure, and it is inhabited by coordinate independent quantities. We often describe that structure and those quantities in a coordinate dependent manner, but the structure of space-time itself is a coordinate independent geometric structure, and the quantities that inhabit space-time are coordinate independent quantities. This coordinate independent structure and those coordinate independent quantities determine what the coordinate dependent representations of that structure and of those quantities look like, and therefore determine how those coordinate dependent representations transform under space-time transformations. That's all there is to it.

Now, what we have just said might seem rather obvious, rather vague, and hence rather useless. However, there are a few important 
lessons to be learned from what we have said that are not always heeded.

Firstly, it means some quantities transform non-trivially (i.e. do not remain identical) under time reversal. (Why it is worth noting this will become clear when we discuss David Albert's views on time reversal.)

Secondly, it means that it is not arbitrary how a quantity transforms under time reversal: how a quantity transforms under time reversal is determined by the (geometric) nature of the quantity in question, not by the absence or presence of a desire to make some theory time reversal invariant. For instance, one might think that one can show that some theory which, prima facie, is not time reversal invariant in fact is time reversal invariant, simply by making a judicious choice for how the fundamental quantities occurring in the theory transform under time reversal. However, if one changes one's view as to what the correct time reversal transformations are for the fundamental quantities occurring in a theory, then one is thereby changing one's view as to the geometric nature of those fundamental quantities, and hence one is producing a new, and different, theory of the world rather than showing that the original theory was time reversal invariant. That is to say, in such a circumstance one faces a choice: this theory with these quantities and these invariances or that theory with those quantities and those invariances. If the competing theories are empirically equivalent then one should make such a choice in the usual manner: on the basis of simplicity, naturalness etc.

In the third place, even if a coordinate dependent formulation of a theory is not invariant under a passive time reversal, this does not yet imply that space-time must have an objective temporal orientation. For coordinate system $x, y, z, t$ and coordinate system $x, y, z, t^{\prime}$ where $t^{\prime}=-t$ not only differ in their temporal orientation, they also differ in their space-time handedness. So failure of invariance of the theory under time reversal need not be due to the existence of an objective temporal orientation, it could be due to the existence of an objective space-time handedness. That is to say, one might be able to form two rival coordinate independent theories, one of which postulates an objective temporal orientation but no space-time handedness, while the other postulates an objective space-time handedness but no temporal orientation. In order to decide which is the better theory, one will have to look at other features of the theories (such as other invariances).

More generally, what we want to know is what structure space-time has, and what quantities characterize the state of its contents. If we have in our possession an empirically adequate coordinate dependent theory, then what we should do is manufacture the best corresponding (i.e. empirically equivalent) coordinate independent theory that we can, and see what space-time structure and what quantities this 
coordinate independent theory postulates. In fact, in the end the issue of what the correct time reversal transformation is is a bit of a red herring. What we are really interested in is what space-time structure there is and what quantities there are (and of course we are interested in the equations that govern their interactions). But the invariances and non-invariances of empirically adequate coordinate dependent formulations of theories are useful for figuring that out.

The above discussion was perhaps a bit abstract. So let us turn to a specific case which has been the subject of a fair amount of debate and controversy, namely that of classical electromagnetism.

\section{Classical electromagnetism: the story so far}

\subsection{The standard textbook view}

Let's start with the standard textbook account of time reversal in classical electromagnetism. The interaction between charged particles and the electromagnetic field is governed by Maxwell's equations and the Lorentz force law. In a particular coordinate system $x, y, z, t$, Maxwell's equations can be written as

$$
\begin{aligned}
\nabla \cdot \mathbf{E} & =\rho \\
\nabla \times \mathbf{E} & =-\frac{\partial \mathbf{B}}{\partial t} \\
\nabla \cdot \mathbf{B} & =0 \\
\nabla \times \mathbf{B} & =\frac{\partial \mathbf{E}}{\partial t}+\mathbf{j},
\end{aligned}
$$

and the Lorentz force law can be written as:

$$
\mathbf{F}=q(\mathbf{E}+\mathbf{v} \times \mathbf{B}) .
$$

Now let us ask how the quantities occurring in these equations transform under time reversal. According to the standard account the active time reverse of a particle that is moving from location $\mathrm{A}$ to location $\mathrm{B}$ is a particle that is moving from $\mathrm{B}$ to $\mathrm{A}$. So, according to the standard view, the ordinary spatial velocity $\mathbf{v}$ must flip over under active time reversal. Obviously, the current $\mathbf{j}$ will also flip over under active time reversal, while the charge density $\rho$ will be invariant under time reversal.

Next let us consider the electric and magnetic fields. How do they transform under time reversal? Well, the standard procedure is simply 
to assume that classical electromagnetism is invariant under time reversal. From this assumption of time reversal invariance of the theory, plus the fact that $\mathbf{v}$ and $\mathbf{j}$ flip under time reversal while $\rho$ is invariant, it is inferred that the electric field $\mathbf{E}$ is invariant under time reversal, while the magnetic field B flips sign under time reversal. Summing up, we have:

$$
\begin{array}{lll}
\mathbf{v} & \stackrel{T}{\longmapsto} & -\mathbf{v} ; \\
\mathbf{j} & \stackrel{T}{\longmapsto} & -\mathbf{j} ; \\
\mathbf{E} & \stackrel{T}{\longmapsto} & \mathbf{E} ; \\
\mathbf{B} & \stackrel{T}{\longmapsto} & -\mathbf{B} ; \\
\rho & \stackrel{T}{\longmapsto} & \rho ; \\
\nabla & \stackrel{T}{\longmapsto} & \nabla ; \\
t & \stackrel{T}{\longmapsto} & -t .
\end{array}
$$

It follows from this time reversal transformation, as straightforward inspection of Maxwell's equations and the Lorentz force law can verify, that time reversal turns solutions into solutions and non-solutions into non-solutions.

\subsection{Albert's proposal}

David Albert ((Albert, 2000), chapter 1) takes issue with the textbooks' account of time reversal in classical electromagnetism. The point of contention is whether or not the magnetic field flips sign under time reversal. The standard account, we have seen, says that it does: $\mathbf{B} \stackrel{T}{\longmapsto}-\mathbf{B}$. Albert suggests, however, that by 'time reversal' one ought to mean 'the very same thing' happening in the opposite temporal order; it follows (according to Albert) that the magnetic field (on a given timeslice) will be invariant under time reversal; and it follows from that (given Maxwell's equations) that the theory is not time reversal invariant. (Albert is happy with a non-trivial time reversal operation for, say, velocity. But that is because velocity is just temporal derivative of position, so of course it flips sign under time reversal. Albert's point is that the magnetic field is not the temporal derivative of anything.)

The difference in direction of argument between Albert and the textbooks is worth highlighting. In the textbooks' account reviewed above, the desideratum that the theory should be time reversal invariant enters as a premise. One finds some transformation on the set of instantaneous states that has the feature that, if it were the time reversal transformation, then the theory would be time reversal invariant, 
and one concludes that this is the time reversal operation. Albert is insisting on the opposite direction of argumentation: one should first work out which transformation on the set of instantaneous states implements the idea of 'the same thing happening backwards in time'; then and only then one should compare one's time reversal operation to the equations of motion, and find out whether or not the theory is time reversal invariant. He is further insisting that, in the case of electromagnetism, this has not adequately been done.

Albert has a point here. One should, indeed, be wary of taking the textbooks' strategy to extremes: it is not difficult to show that, under very general conditions, any theory, including ones that are (intuitively!) not time reversal invariant, can be made to come out 'time reversal invariant' if we place no constraints on what counts as the 'time reversal operation' on instantaneous states. ${ }^{1}$

So something in Albert's objection seems to be right. We do not, however, endorse his account of time reversal in electromagnetism. We will come back to this after discussing an alternative account, due to David Malament.

\subsection{Malament's proposal}

Malament seeks to 'justify' the usual textbook time reversal operation for classical electromagnetism, and for the $\mathbf{B}$ field in particular.

At first sight, one might think that this is done as soon as one thinks relativistically, and conceives of the $\mathbf{E}$ and $\mathbf{B}$ fields as components of the Maxwell-Faraday tensor $F^{a b}$. A moment's thought, however, shows that this is not the case. The electric field is read off from the spacetime components of $F^{a b}$, while the magnetic field is read off from the

\footnotetext{
${ }^{1}$ Here is an example. Suppose that we have a single particle in one dimension. Let $r$ denote its position; let its instantaneous state space be given by $(0, \infty]$. Let its equation of motion be given by$$
\frac{d r}{d t}=e^{-k r}
$$

where $k$ is a positive constant. This theory is (intuitively) not time reversal invariant: it says that the particle's position coordinate always decreases. However, if we are really willing to let the time reversal operation be whatever is required to secure time reversal invariance, the intuition of asymmetry can easily be violated: simply let the time reversal operation be $r \mapsto \frac{1}{r}$.
} 
space-space components: ${ }^{2}$

$$
F^{\mu \nu}=\left(\begin{array}{cccc}
0 & E_{1} & E_{2} & E_{3} \\
-E_{1} & 0 & B_{1} & -B_{2} \\
-E_{2} & -B_{3} & 0 & B_{1} \\
-E_{3} & B_{2} & -B_{1} & 0
\end{array}\right) .
$$

If the Maxwell-Faraday tensor $F^{a b}$ itself (as a tensor) is invariant under time reversal, then it will be the electric field, not the magnetic field, that flips sign when we perform a passive time reversal. To justify the standard textbook transformation, we need to justify a sign flip for $F^{a b}: F^{a b} \stackrel{T}{\longmapsto}-F^{a b}$. This is the task that Malament takes up.

Malament's treatment of electromagnetism embodies a particular conception of what it means to 'justify' a time reversal operation, and, relatedly, a third conception (alongside active and passive time reversal) of what time reversal is. We will first state these explicitly (but somewhat abstractly), then let our exposition of Malament's treatment of electromagnetism illustrate them:

- To give a justification of a non-trivial time reversal operation $X \stackrel{T}{\longmapsto} X^{\prime}$ for a state description $X$ is to postulate a particular fundamental ontology for the theory, and to explain how the representation relation between $X$ and the objects of the fundamental ontology depends on temporal orientation, in such a way that it follows that if we flip the temporal orientation but hold the remainder of the fundamental objects fixed, the state description changes as $X \stackrel{T}{\longmapsto} X^{\prime}$.

- Geometric time reversal: To time-reverse a kinematically possible world, hold all the fundamental quantities fixed [with the exception of the temporal orientation, if that is a fundamental object], and flip the temporal orientation.

Malament's treatment of electromagnetism. Malament's account is as follows. There are two fundamental types of objects in a classical electromagnetic world. There are the world-lines of charged particles, and there is the electromagnetic field. Now, the dynamics happens to be such that it will be convenient, mathematically, to represent the motions of particles by means of four-velocities, where the four-velocity at any point on the worldline is tangent to the worldline at that point. The crucial fact now is that a world-line does not have a unique tangent vector at a point: at each point on a world-line, there is

\footnotetext{
${ }^{2}$ Roman subscripts and superscripts indicate that we are using the abstract index notation: $F^{a b}$ is a rank two tensor, not a component of such a tensor in a particular coordinate system. When we wish to refer to coordinate-dependent components of tensors, we use Greek indices, as in $F^{\mu \nu}$.
} 


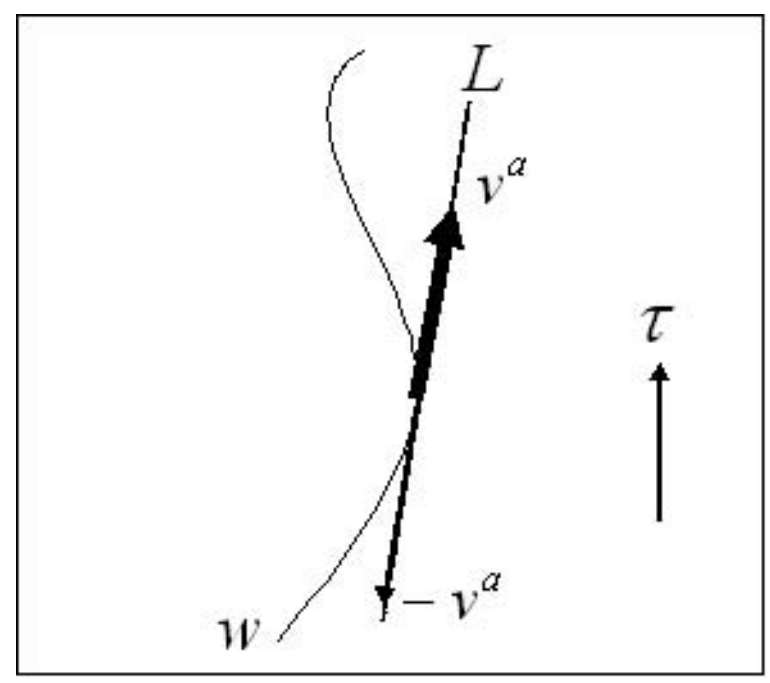

Figure 1: $w$ is the worldline of a particle of mass $m$ and charge $q . \quad L$ is the tangent line to $w$ at the point $p$. Until we have specified a temporal orientation $\tau$, the four-velocity could be $v^{a}$ or $-v^{a} \cdot v^{b} \nabla_{b} v^{a}$ is the fouracceleration; it is independent of temporal orientation. The electromagnetic field $F$ maps $\langle L, q\rangle$ to the four-force $m v^{b} \nabla_{b} v^{a}$.

a continuous infinity of four-vectors that are tangent to the world-line at the point in question. We can narrow things down somewhat by stipulating that four-velocities are to have unit length, but this still does not quite do the trick: one can associate two unit-length fourvectors that are tangent to the world-line at the point in question (if $v^{a} \in T_{p}$ is one, then $-v^{a}$ is the other; see figure 1).

Next, how should we conceive of an electromagnetic field at a point $p$ in spacetime? According to Malament, we should think of the electromagnetic field at $p$ as a quantity which, for any tangent line $L$ at $p$ and charge $q$, determines what 4 -force a (test) particle with charge $q$ and tangent line $L$ at $p$ would experience. More formally, Malament conceives of the electromagnetic field $F\left(\right.$ not $\left.F^{a b}\right)$ at a point $p$ as a map from pairs $\langle L, q\rangle$ at $p$ to four-vectors at $p$.

How do Malament's fundamental quantities (tangent lines, maps from tangent lines to 4-vectors) relate to the standard fundamental quantities (4-vectors, Maxwell-Faraday tensor), the ones occurring in our three equations? The relation is simple: relative to a choice of temporal orientation, one can associate a unique unit-length tangent vector with each location on a timelike world-line, namely, the one that is 'future'-directed according to that temporal orientation. So, 
given a temporal orientation, we can represent any given tangent lines by a unique unit length four-vector, i.e. four-velocities. Given such a representation, the electromagnetic field can be represented by a linear map from four-vectors to four-vectors. And that just means that, given a temporal orientation we can represent the electromagnetic field as a rank 2 tensor, which we can identify as the standard representation of the electromagnetic field by the Maxwell-Faraday tensor $F^{a b}$.

So, given a temporal orientation, Malament can formulate classical electromagnetism using the usual covariantly-formulated equations: the Maxwell equations,

$$
\begin{gathered}
\nabla_{[a} F_{b c]}=0 \\
\nabla_{n} F^{n a}=J^{a},
\end{gathered}
$$

and the Lorentz force law,

$$
q F^{a}{ }_{b} V^{b}=m V^{b} \nabla_{b} V^{a} .
$$

Using the geometric conception of time reversal, it is then straightforward to see how the quantities in these equations transform under time reversal. Recall that on the geometric conception, to 'time reverse' is to leave all the fundamental quantities fixed, and to flip temporal orientation. We then hold fixed (also) our conventions about how non-fundamental quantities are derived from the fundamental ones in an orientation-relative way, and we see what transformations for the non-fundamental quantities result. Now, on Malament's picture, fourvelocity is not fundamental: it is defined only relative to a choice of temporal orientation. If $v^{a}$ is the four-velocity, i.e. is the unit-length future-directed tangent, to a given worldline at some point $p$ relative to our original choice of temporal orientation, then $-v^{a}$ will be the four-velocity relative to the opposite choice of temporal orientation. Similarly, if $F^{a b}$ correctly maps four-velocities four-forces relative to our original orientation, then, in order to represent the same map from tangent lines to four-forces relative to the opposite choice of temporal orientation, we will have to flip the sign of the tensor, to compensate for the sign flip in four-velocity: $F^{a b} \mapsto-F^{a b}$. We have now given justifications for Malament's time reversal operations for $v^{a}$ and $F^{a b}$ :

$$
\begin{array}{ccc}
v^{a} & \stackrel{T}{\longmapsto} & -v^{a} ; \\
F^{a b} & \stackrel{T}{\longmapsto} & -F^{a b} .
\end{array}
$$

Electric and magnetic fields. As Malament notes, the frameindependent formulation suffices to write down the dynamics of the theory and establish their time-reversal invariance. Like Malament, 
however, we wish to make contact with Albert and the textbooks; to do this, we need to consider decompositions of our four-dimensional $F^{a b}$ into electric and magnetic fields.

Following Malament ((2004):pp.16-17), we make the following two definitions:

- A volume element $\epsilon_{a b c d}$ on $M$ is a completely antisymmetric tensor field satisfying the normalization condition $\epsilon_{a b c d} \epsilon^{a b c d}=$ -24 .

- A frame $\eta_{a}$ is a future-directed, unit, timelike vector field that is constant $\left(\nabla_{a} \eta^{b}=0\right)$.

We can now decompose the electromagnetic field into electric and magnetic fields, relative to a given frame and volume element:

$$
\begin{aligned}
E^{a} & :=F^{a}{ }_{b} \eta^{b} ; \\
B^{a} & :=\frac{1}{2} \epsilon^{a b c d} \eta_{b} F_{c d} .
\end{aligned}
$$

Note that the electric field $E^{a}$ and is defined relative to temporal orientation and frame; the magnetic field $B^{a}$ is defined relative to temporal orientation, frame and volume element. The volume element itself is a more subtle case; we follow Malament in stipulating that it, too, flips sign under time reversal. ${ }^{3}$

It follows that (as Malament explains) the time reversal transformation acts as follows:

$$
\begin{aligned}
& \tau \stackrel{T}{\longmapsto}-\tau \text {; } \\
& \eta_{a} \stackrel{T}{\longmapsto}-\eta_{a} ; \\
& \epsilon_{a b c d} \stackrel{T}{\longmapsto}-\epsilon_{a b c d} ; \\
& v^{a} \stackrel{T}{\longmapsto}-v^{a} ;
\end{aligned}
$$

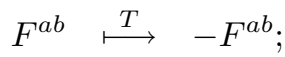

$$
\begin{aligned}
& E^{a} \stackrel{T}{\longmapsto} E^{a} ; \\
& B^{a} \stackrel{T}{\longmapsto}-B^{a} .
\end{aligned}
$$

Note that the electric field, $E^{a}$, is invariant under time reversal, while the magnetic field, $B^{a}$, flips sign. This is exactly the time reversal operation suggested by standard textbooks in classical electromagnetism. So, Malament's proposal provides a justification, based on his geometric conception of time reversal, for the standard view.

\footnotetext{
${ }^{3}$ The point here is just that we choose to mean, by 'time reversal', 'flip the temporal orientation and hold the spatial handedness fixed' (so the total orientation, represented by the sign of the volume element, has to flip), rather than 'flip the temporal orientation and hold the total orientation fixed' (in which case the spatial handedness would have to flip).
} 


\subsection{Albert revisited}

We noted that, as soon as one thinks of the $\mathbf{E}$ and $\mathbf{B}$ fields as derived from a more fundamental Maxwell-Faraday tensor, either $\mathbf{E}$ or $\mathbf{B}$ must flip sign under time reversal. On Albert's account, neither flips sign. But, of course, Albert is perfectly aware of the four-dimensional formulation of electromagnetism. So why does he say what he says?

Well, on Albert's view, pace any arguments for interpreting electromagnetism in terms of a Minkowski spacetime, spacetime is in fact Newtonian, velocities are good old spatial 3 -vectors, and so are the electric and magnetic fields. The dynamics governing the development of the $\mathbf{E}$ and $\mathbf{B}$ fields, and the particle worldlines, happens to be 'pseudoLorentz invariant': that is, there exist simple transformations on the $\mathbf{E}$ and $\mathbf{B}$ fields such that, if those were the ways $\mathbf{E}$ and $\mathbf{B}$ transformed under Lorentz transformations, then the theory would be Lorentz invariant. This is perhaps surprising - there's no a priori reason to expect the dynamics to have this feature of 'pseudo Lorentz invariance', if one thinks that spacetime is Newtonian. But then, there's no a priori reason why the dynamics in a Newtonian spacetime shouldn't be pseudo Lorentz invariant, either. Similarly: it follows from this pseudo Lorentz invariance that observers will never be able to discover, merely by means of 'mechanical experiments' (observations of particle worldlines), what their absolute velocity is, or pin down the $\mathbf{E}$ and $\mathbf{B}$ fields uniquely. So if one thought that all features of reality must be empirically accessible to the human machine with its coarsegrained perceptive capacities, one would be very suspicious of Albert's view; but why, Albert might well ask in reply, should one think that?

What should one make of all this? Well, while we agree that Albert's view is internally coherent, we regard it as insufficiently motivated, for the following reason. A straightforward application of Ockham's razor prescribes that, faced with a choice between two empirically equivalent theories, one of which is strictly more parsimonious than the other as far as spacetime structure goes, one should (ceteris paribus) prefer the more parsimonious theory. In other words, one should commit to the minimum amount of spacetime structure needed to account for the empirical success of our theories. Now, on Albert's view, spacetime is equipped with a preferred foliation and a standard of absolute rest; further, it must also be equipped with an objective temporal orientation, in order to account for the non-timereversal invariance of classical electromagnetism. On the Minkowskian view, spacetime has none of this structure. If other things are equal, this gives us a reason to prefer a Minkowskian view; further, as far as we can see, other things are equal. We conclude that, insofar as classical electromagnetism is to be trusted at all, spacetime is Minkowskian rather than Newtonian, it is the unified electromagnetic field, rather 
than the $\mathbf{E}$ and $\mathbf{B}$ fields separately, that is fundamental, and that $\mathrm{Al}-$ bert's view of time reversal is false.

We will say no more about Newtonian interpretations. What is more interesting, for the purposes of our paper, is that even given a Minkowskian interpretation of relativity, the ontology, and hence the time reversal operation, for classical electromagnetism remains underdetermined. Malament has suggested one candidate ontology; we turn now to alternatives.

\section{The 'Feynman' proposal}

In this section, we turn to the view of time reversal that will correspond to Feynman's view of antiparticles. Our discussion here will not differ from our discussion of Albert's or Malament's proposals in terms of what time reversal is or how non-trivial time reversal operations are justified; that is, we are still thinking in terms of geometric time reversal. The 'Feynman' proposal is simply a different proposed ontology, a different view as to what fundamental quantities there in fact are out there in nature. It provides an geometric justification for a third time reversal operation for the electric and magnetic fields, distinct from both Albert's and Malament's.

Fundamental ontology. The distinctive feature of the 'Feynman' proposal is the suggestion that there is a fundamental, temporal orientation-independent fact as to the sign of the four-velocity of a given particle. The ontology is not one of worldlines and an electromagnetic field; it is one of directed worldlines and an electromagnetic field. In that case, we no longer have Malament's motivation for saying that the electromagnetic field is a map from tangent lines to fourvectors. So, on the 'Feynman' proposal, we take the electromagnetic field to be (fundamentally!) a map from four-vectors to four-vectors, or, equivalently, a rank 2 tensor field. Thus, the electromagnetic field, independent of a temporal orientation, corresponds to a unique rank 2 tensor: the Maxwell-Faraday tensor $F^{a b}$.

The electric and magnetic fields, $E^{a}$ and $B^{a}$, are then defined from $F^{a b}$, relative to a frame and volume element, just as they are on Malament's proposal.

Time reversal. The corresponding time reversal transformation is:

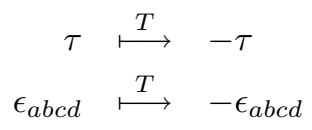




$$
\begin{array}{ccc}
\eta^{a} & \stackrel{T}{\longmapsto} & -\eta^{a} \\
F^{a b} & \stackrel{T}{\longmapsto} & F^{a b} \\
v^{a} & \stackrel{T}{\longmapsto} & v^{a} \\
E^{a} & \stackrel{T}{\longmapsto} & -E^{a} \\
B^{a} & \stackrel{T}{\longmapsto} & B^{a} .
\end{array}
$$

Note that this is not the textbook time-reversal transformation. The Feynman proposal has the consequence that the electric field flips sign under time reversal, and that the magnetic field does not - but it, too, has the consequence that the theory is time reversal invariant. ${ }^{4}$

More on the 'Feynman' proposal. Certain features of the time-reversal operation sanctioned by the 'Feynman' proposal seem rather odd, however; let's take a closer look. Consider, for example, a particle travelling between Harry and Mary (see figure 2). Suppose that, prior to time reversal, the particle's four-velocity happens to be 'future'-directed, and points from Harry's worldline to Mary's. Then, the following two observations can be made about the timereversed situation. First, in the time-reversed situation the particle's four-velocity will be 'past'-directed. (This follows from the fact that the four-velocity itself dows not change, while the description of a given temporal direction on the manifold as 'future'/ 'past' does change when we flip the temporal orientation.) Second, the four-velocity will still point from Harry to Mary. On the 'Feynman' proposal, that is, we are asked to make sense of a notion of 'time reversal' according to which the time-reverse of a particle traveling from Harry to Mary is not a particle traveling from Mary to Harry. This seems an odd feature of the 'Feynman' view.

However, let us suppose that it is not the case that the fourvelocities of all particles point in the same temporal direction. That is, let us suppose that, relative to a fixed choice of temporal orientation, some particles have future-directed four-velocities, and others have past-directed four-velocities. Suppose then that we have a model of electromagnetism which consists of a single particle of charge $q$ moving in an electromagnetic field: $\left\langle F^{a b},\left\langle v^{a}, q\right\rangle\right\rangle$. One can then trivially produce another model by keeping the electromagnetic field $F^{a b}$ the

\footnotetext{
${ }^{4}$ The time reversal invariance of this theory is easy to see, by looking at the Lagrangian $L=-\frac{1}{4} F_{a b} F^{a b}-q V_{a} A^{a}$. Under 'Feynman' time reversal, all four of the objects appearing in this Lagrangian - the Maxwell-Faraday tensor $F^{a b}$, the charge $q$, the four-velocity $v^{a}$ and the four-potential $A^{a}$ - are invariant under time reversal. So of course the Lagrangian itself (a scalar field on $M$ ) is invariant under time reversal, and, consequently, there will never be a set of field configurations and particle worldlines that is dynamically permitted relative to one temporal orientation and not the other.
} 


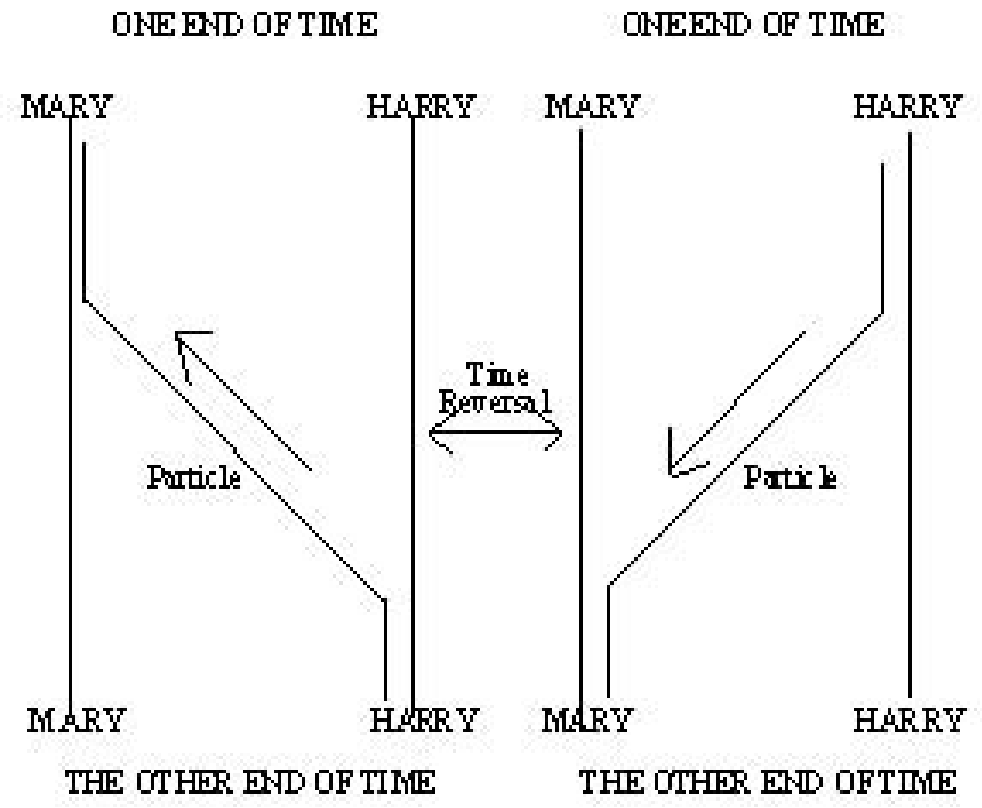

Figure 2: The time-reverse of a particle traveling from Mary to Harry, according to the Feynman view, is (still) a particle traveling from Mary to Harry. 
same and the trajectory the same, while flipping the sign of the charge and of the four-velocity: $\left\langle F^{a b},\left\langle-v^{a},-q\right\rangle\right\rangle$. (One can see that this operation does indeed turn models into models by inspecting Maxwell's equations and the Lorentz force law, or, alternatively, by inspecting the Lagrangian. The only changes in any of these quantities are in the signs of $q$ and $v^{a}$, which always occur together, so that the changes cancel; so, changing the sign of the charge and of the four-velocity must turn a solution into a solution, and a non-solution into a non-solution.)

Let us put this another way: a particle with charge $q$ and fourvelocity $v^{a}$ behaves, in a given electromagnetic field, exactly as if it is a particle with charge $-q$ and velocity $-v^{a}$ : it follows exactly the same trajectory, so that, given only access to the results of 'mechanical experiments', the two possible situations cannot be distinguished in any way. This observation opens the door for the following hypothesis: particles that we have regarded as belonging to different types, related by the 'is the antiparticle of' relation - electrons and positrons, say - are really of the same type as one another. In particular, they have the same electric charge as one another. Things appear otherwise only if we erroneously assume that all four-velocities must point in the same temporal direction as one another. In other words, we can achieve parsimony in particle types at the cost of the 'extravagance' of endowing particle worldlines with an intrinsic direction; the Feynman proposal is that we do so. If this hypothesis is right, then it is indeed true that an anti-particle is nothing but a particle traveling in the opposite direction of time.

\section{Structuralism: A Third Way}

We have been assuming so far that the Malament and Feynman proposals represent distinct alternatives, at most one of which can be correct. One can have a different time reversal operation for the same formalism, we said, only if one makes a different postulate about the fundamental ontology; but if one does that, then one has changed one's theory, in the clear sense that one has changed one's hypothesis about the fundamental nature of the world.

Be that as it may, one might still (on the other hand) have the gut feeling that the 'disagreement' between the Malament and Feynman 'ontologies' is not a serious one; that the two 'rival theories' are, in some sense, saying the same thing in different ways.

Clearly, one cannot fully hold onto both of these ideas: one says that the Malament and Feynman proposals are distinct, the other says they

are not. In the present section, however, we will sketch a third set of hypotheses about the fundamental nature of a classical electromagnetic world that does justice to the basic principles behind both ideas. It will 
do justice to the just-mentioned gut feeling, in that it will provide a way of regarding the claim that worldlines have arrows on them and that four-velocities can be past-directed (as Feynman says), and the claim that worldlines have no intrinsic arrows and four-velocities are always future directed (as Malament says), as equivalent descriptions of the same underlying situation. However, it will also do justice to our earlier insistence that this business of formulating alternative descriptions is not ontologically innocent, because it will be a third, rival, suggestion for what the fundamental nature of electromagnetic reality might be, rather than a claim that the original Malament and Feynman theories are equivalent.

To set out our third alternative, let us first define 'Malament picture' and 'Feynman picture'. A Malament picture is a world-description according to which:

- all four-velocities are future-directed;

- worldlines fall into natural equivalence classes, where the members of a given equivalence class have the same absolute value of charge as one another;

- each equivalence class subdivides into two, where the charge of the members of one sub-class has the opposite sign to the charge of the members of the other sub-class.

A Feynman picture is a world-description in which:

- four-velocities can be future- or past-directed;

- worldlines fall into natural equivalence classes, where the members of a given equivalence class have the same charge as one another;

- each equivalence class subdivides into two, where the four-velocities of the members of one subclass point in the opposite temporal direction to the four-velocities of the members of the other subclass.

Then, the structuralist idea is that there is an underlying reality which can be represented by either a Malament or a Feynman picture, and that the choice between the two pictures is a conventional one (i.e. both types of picture do an equally good job of representing the underlying facts).

We proceed as follows. Suppose that neither the charge nor the four-velocity direction is an intrinsic property of worldlines. Suppose that what is fundamental, in our electromagnetic world, is a set $W$ of worldlines, and a function $f . f: W \times W \rightarrow R$ is a function from ordered pairs of worldlines to real numbers, with the following two properties:

$$
\forall w_{i}, w_{j}, w_{k} \in W, \quad f\left(w_{i}, w_{j}\right) \cdot f\left(w_{j}, w_{k}\right)=f\left(w_{i}, w_{k}\right) ;
$$




$$
\forall w \in W, \quad f(w, w)=1 .
$$

(Intuitively: in terms of Malament pictures, the interpretation of this function is that $f\left(w_{i}, w_{j}\right)=\frac{q_{i}}{q_{j}}$, i.e. the ratio of the charge of worldline $w_{i}$ to that of $w_{j}$.)

To build a Malament picture given $W$ and $f$, we proceed as follows:

1. Arbitrarily select a worldline $w$ from the set $W$.

2. Arbitrarily select a real number $q$ to represent this particle's charge.

3. Use the function $f(w, \cdot)$ to fix the values of the charges of all the other particles: the charge of worldline $w^{\prime}$ is given by $q \cdot f\left(w, w^{\prime}\right)$.

4. Pick a temporal orientation, and stipulate that all four-velocities are to be future-directed relative to that choice of temporal orientation.

It follows from this construction, in the usual way, that four-velocities flip sign under geometric time reversal.

Alternatively, to build a Feynman picture to represent the same structuralist world, we adopt a slightly different procedure:

1. Arbitrarily select a worldline $w$ from the set $W$.

2. Arbitrarily select a positive real number $q$ to represent this particle's charge.

3. Arbitrarily select a temporal direction for the four-velocity of $w$.

4. Use the function $f(w, \cdot)$ to fix the values of the charges of all the other worldlines: the charge of worldline $w^{\prime}$ is given by $q$. $\left|f_{1}\left(w, w^{\prime}\right)\right|$.

5. Use the function $f(w, \cdot)$ to determine the temporal directions of the four-velocities of all other worldlines: if $f\left(w, w^{\prime}\right)>0$, the four-velocity of $w^{\prime}$ is to point in the same temporal direction as that of $w$; if $f\left(w, w^{\prime}\right)<0$, the four-velocity of $w^{\prime}$ is to point in the opposite temporal direction as that of $w .^{5}$

6. If desired, pick a temporal orientation, and say that all worldlines whose four-velocities are future-directed are particles, those whose four-velocities are past-directed antiparticles.

It follows from this construction, as before, that nothing except our designation of worldlines as 'particles' or 'antiparticles' changes under geometric time reversal.

In this way, it can be a consequence of our third candidate ontology that Malament-pictures and Feynman-pictures are merely alternative conventions, and that answers to questions like whether or not fourvelocity flips sign under time reversal, whether time reversal turns particles into antiparticles, and so on, are convention-dependent.

\footnotetext{
${ }^{5}$ We ignore the possibility of neutral particles.
} 


\section{Concluding remarks}

In this final section, we summarize our conclusions to date, and then indicate some open issues that we would like to resolve.

We have elaborated the 'geometric' notion of time reversal introduced by Malament, according to which time reversal consists in leaving all [other] fundamental quantities alone, and merely flipping the temporal orientation. This allows us to give an account, as the passive and active notions of time reversal cannot, of how it may come about that a coordinate-independent quantity such as $F_{a b}$ transforms nontrivially under time reversal. We have then discussed four approaches to time reversal in classical electromagnetism in the light of this geometric conception: Albert's, Malament's, the 'Feynman' approach, and the structuralist approach. Only according to Albert is the theory not time reversal invariant; we have rejected Albert's account by appeal to Ockham's Razor.

How one should go about choosing between the Malament, Feynman and Structuralist ontologies, we are not sure. Our feeling is that Structuralism is preferable because it eliminates distinctions that seem to be devoid of differences. But it would be better if this 'feeling' could be replaced with argument. One possibility is that there is an argument favoring this sort of structuralism in general, without regard to the specific details of classical electromagnetism. Another is that viewing classical electromagnetism as the classical limit of a quantum field theory may introduce considerations that favor one ontological position over others. The investigation of these possibilities is a future project.

Suppose, though, that Structuralism is indeed correct. Then the difference between the Malament and Feynman languages is just that - a difference in language; one's choice of language is a convention. In the case of classical electromagnetism, not much hangs on the choice of convention, since the theory comes out time reversal invariant according to both Malament and Feynman. A more interesting case would be one in which the time reversal invariance of the theory was (according to structuralism) a convention-dependent matter. Given the standard link between spacetime symmetries and spacetime structure, this would render the question of whether or not a privileged temporal orientation exists a convention-dependent matter. It is not immediately clear whether this makes sense, or what conclusion one should draw if it doesn't.

A final remark on field theories is in order. The original motivation for this project was the feeling that the existence of a CPT theorem is rather puzzling - why should charge conjugation be so intimately related to spacetime symmetries? The point here is that, according to the 'Feynman' proposal, the operation that ought to be called 'time reversal' - in the sense that it bears the right relation to spatiotem- 
poral structure to deserve that name - is the operation that is usually called $T C$; on this proposal, the theorem known as the 'CPT theorem' would be more properly called a PT theorem. Our next project is to investigate a geometrical understanding of the (classical and quantum

field-theoretic) 'CPT' theorems, drawing on the ideas outlined in this paper.

\section{References}

Albert, D. (2000). Time and chance. Cambridge, MA: Harvard University Press.

Feynman, R. (1985). Qed. Princeton: Princeton University Press.

Malament, D. (2004). On the time reversal invariance of classical electromagnetic theory. Studies in History and Philosophy of Modern Physics, 35B(2), 295-315. 